\title{
LES ÉLECTIONS MUNICIPALES ET AUTONOMIQUES DU 22 MAI 2011 EN ESPAGNE ET EN CATALOGNE
}

\author{
Joan Marcet et al.
}

ARPoS | Pôle Sud

$2011 / 2-n^{\circ} 35$

pages 130 à 143

ISSN 1262-1676

Article disponible en ligne à l'adresse:

http://www.cairn.info/revue-pole-sud-2011-2-page-130.htm

Pour citer cet article :

Marcet Joan et al., « Les élections municipales et autonomiques du 22 mai 2011 en Espagne et en Catalogne », Pôle Sud, 2011/2 n³5, p. 130-143.

Distribution électronique Cairn.info pour ARPoS.

(c) ARPoS. Tous droits réservés pour tous pays.

La reproduction ou représentation de cet article, notamment par photocopie, n'est autorisée que dans les limites des conditions générales d'utilisation du site ou, le cas échéant, des conditions générales de la licence souscrite par votre établissement. Toute autre reproduction ou représentation, en tout ou partie, sous quelque forme et de quelque manière que ce soit, est interdite sauf accord préalable et écrit de l'éditeur, en dehors des cas prévus par la législation en vigueur en France. II est précisé que son stockage dans une base de données est également interdit. 


$$
\begin{aligned}
& \text { Chroniques } \\
& \text { Électorales }
\end{aligned}
$$




\title{
LES ÉLECTIONS MUNICIPALES ET AUTONOMIQUES DU 22 MAI 2011 en Espagne et en Catalogne
}

\author{
Joan Marcet \\ Oriol Bartomeus
}

Universitat Autònoma de Barcelona

Institut de Ciències Polítiques i Socials

Traduction: Emmanuel Négrier

\section{RÉSUmé / AbStRAct}

Les élections municipales et autonomiques qui se sont déroulées le 22 mai 2011 en Espagne représentent le plus important succès électoral du Parti Populaire à l'échelle territoriale. Ce papier analyse les données du scrutin, en observant la participation électorale, la répartition territoriale des votes obtenus par chacune des formations politiques, de même que la position de ces élections municipales et autonomiques dans le cycle électoral des vingt dernières années. Après avoir traité des explications globales de ce renversement, il se focalise sur la Catalogne, où seules avaient lieu les élections municipales.

Municipal and autonomic elections, which took place in May 2011 in Spain, represent the largest electoral victory for the Popular Party at the territorial level. This paper analyzes data polling, scrutinizing voters' turnout, the territorial distribution of electoral votes obtained by each party as well as the position of the autonomic and municipal elections within the electoral cycle of the last twenty years. After dealing with global explanations of these results, it focuses on Catalonia, where only the municipal elections were held.

\section{MOTS-CLÉS / KEYWORDS}

Catalogne, crise économique, cycle électoral, élections, Espagne

Catalonia, economic crisis, election cycle, elections, Spain 
Les élections municipales et autonomiques qui se sont déroulées le 22 mai 2011 en Espagne représentent le plus important succès électoral du Parti Populaire à l'échelle territoriale ; ce qui, joint à sa victoire annoncée lors des élections législatives de novembre 2011, lui accorde pouvoir et contrôle des différentes échelles de gouvernement à un niveau jamais atteint.

La victoire du PP, qui a été qualifiée de renversement électoral (Urquizu, 2011), bien qu'elle ait été pronostiquée par tous les sondages, est liée à une pluralité de motivations et d'explications, de la conjoncture de grave crise économique que supporte le pays depuis trois ans, jusqu'à la détérioration de l'image et de la crédibilité du gouvernement socialiste présidé par Rodríguez Zapatero, l'une des conséquences de sa gestion de ladite crise; de la crise politique qui couvait et a émergé au cours de la dernière période - et dont le mouvement du 15-M ou de "Los Indignados" n'est qu'un indice - jusqu'au déclin électoral et la désorientation politique de la gauche espagnole, et en particulier celle du Parti Socialiste.

Avant d'approfondir quelques uns des éléments qui peuvent contribuer à expliquer les résultats des élections du 22 mai, il faut examiner les données de ce scrutin, en analysant la participation électorale, la répartition territoriale des votes et du capital électoral obtenu par chacune des formations politiques, de même que la position de ces élections municipales et autonomiques dans le cycle électoral des vingt dernières années. Nous nous réfèrerons en premier lieu aux résultats électoraux dans l'ensemble de l'Espagne, puis nous nous centrerons sur la Catalogne, où seules eurent lieu les élections municipales, tout comme en Pays Basque, Galice et Andalousie, des communautés qui disposent de leur propre calendrier autonomique.

Tableau 1. Résultats des élections municipales en Espagne (1991-2001), en suffrages exprimés

\begin{tabular}{|l|c|c|c|c|c|c|}
\cline { 2 - 7 } \multicolumn{1}{c|}{} & 1991 & 1995 & 1999 & 2003 & 2007 & 2011 \\
\hline Participation & 62,78 & 69,87 & 63,99 & 67,67 & 63,97 & 66,23 \\
\hline Votes nuls & 0,7 & 0,68 & 0,91 & 1,3 & 1,17 & 1,7 \\
\hline Votes blancs & 1,13 & 1,46 & 1,95 & 1,76 & 1,92 & 2,54 \\
\hline \multicolumn{7}{|l|}{} \\
\hline P.P. & 25,34 & 35,27 & 34,44 & 34,29 & 35,62 & 37,53 \\
\hline P.S.O.E.-P.S.C. & 38,34 & 30,84 & 34,26 & 34,83 & 34,92 & 27,79 \\
\hline I.U. - ICV/EUiA & 8,38 & 11,68 & 7,6 & 7,53 & 6,64 & 7,43 \\
\hline CiU & 4,86 & 4,39 & 3,63 & 3,44 & 3,25 & 3,45 \\
\hline ERC & 0,49 & 0,92 & 1,06 & 1,83 & 1,56 & 1,2 \\
\hline PNV & 1,59 & 1,41 & 1,28 & 1,78 & 1,39 & 1,45 \\
\hline BILDU/EH/HB & 1,06 & 0,83 & 1,28 & & & 1,39 \\
\hline BNG & 0,57 & 0,94 & 1,36 & 1,42 & 1,42 & 1,16 \\
\hline
\end{tabular}

Source : Élaboration propre d'après données du Ministère de l'Intérieur Abréviations :

P.P.

P.S.O.E.-P.S.C

I.U. - ICV/EUIA

CiU

ERC

PNV

BILDU/EH/HB

BNG
Parti Populaire, (droite)

Parti Socialiste Ouvrier Espagnol, Parti Socialiste de Catalogne

Gauche Unie, Initiative pour Catalogne Verts, Gauche Unie et Alternative

Convergence et Union (centre droit nationaliste catalan)

Gauche Républicaine de Catalogne

Parti Nationaliste Basque

Coalition indépendantiste basque

Bloc Nationaliste Galicien 


\section{LA PARTICIPATION ÉLECTORALE}

La participation aux élections municipales a atteint $66,23 \%$ des suffrages exprimés, soit $2,26 \%$ de plus que lors des précédentes, ce qui traduit une participation moyenne sur la période 1987-2007. Ce regain de participation s'est produit dans toutes les communautés autonomes - à l'exception de la Navarre, où elle a baissé de 3,5 points - même dans celles où il n'y avait pas d'élection autonomique.

Cette participation croissante peut s'interpréter comme le surcroît de mobilisation électorale qui précède tout changement de majorité, comme on l'a déjà vu en 1995 avec ensuite la victoire du PP de 1996, ou en 2003 et celle du PSOE de 2004. En effet les élections municipales ont toujours connu un niveau de participation inférieur à celui des élections législatives, mais en 2003 et encore plus en 1995, lorsque cette participation avoisina les $70 \%$, l'augmentation de la mobilisation électorale sembla anticiper la victoire électorale du parti de gouvernement concurrent au niveau national (Delgado, 1997).

La participation est également favorisée par la simultanéité des scrutins dans 13 communautés autonomes, ainsi que par l'existence d'une confrontation majeure dans quelques unes d'entre elles. Lors des élections du 22 mai, les communautés où la participation fut la meilleure furent Castille-La Manche (76,7 \%) et Extremadure (76,3\%), où s'affrontaient un PSOE sortant et un PP dans l'opposition depuis 1983. La possibilité d'un changement majeur à l'échelle régionale a contribué à renforcer la participation d'un électorat déjà traditionnellement participatif.

À l'échelle municipale, on peut parler d'une certaine remobilisation urbaine, comme l'a signalé Irène Delgado, en se référant au regain de participation de 1995 (Delgado, 2010). Dans la quasi totalité des grandes villes, la participation a dépassé celle de 2007, en dépit du fait que cette participation est fonction de la taille des municipalités. Plus celle-ci baisse, plus la participation est forte ; la plus grande proximité politique et un contact plus régulier avec l'administration municipale semble favoriser la participation. Comme nous le verrons ensuite, ce regain coïncide, dans les grandes villes, avec celui du vote pour le Parti Populaire (ou pour CiU en Catalogne, et PNV en Pays Basque), ce qui témoigne d'une mobilisation plus élevée de l'électorat de centre-droit.

\section{LE RÉSULTAT DES ÉLECTIONS, ET LA RÉPARTITION TERRITORIALE DU POUVOIR MUNICIPAL ET RÉGIONAL}

Comme nous l'avons déjà indiqué, le clair vainqueur de ces élections est le Parti Populaire, qui a dépassé de 10\% le score du Parti Socialiste. Le PP a triomphé en voix et en nombre de conseillers, un fait que ne s'était pas produit lors du scrutin de 2007 : s'il avait dépassé le PSOE en voix, il s'était incliné devant lui en nombre total de sièges. 
En ce qui concerne les élections autonomiques, le PP atteint pratiquement les 9 millions de voix, soit un score supérieur de $7 \%$ à celui des socialistes. Le PP est le premier parti dans des communautés jusque là gouvernées par le PSOE, comme Castille La Manche, Estrémadure et Aragon. Il n'y a qu'en Navarre et en Asturies que la situation est spécifique. Dans le premier cas, c'est l'Unión del Pueblo Navarro (UPN) qui l'emporte. Il s'agit de la "marque" électorale du PP dans cette communauté jusqu'en 2009. Dans les Asturies, c'est une dissidence du même PP, le Foro Asturias Ciudadano (FAC) dirigé par l'ancien secrétaire général du Parti Populaire, Francisco Álvarez Cascos, qui obtient la victoire. Dans toutes les autres communautés où les élections autonomiques avaient lieu, le PP consolide son hégémonie, y compris dans des communautés comme celle de Valence, où il affronte de redoutables problèmes de corruption depuis quelques années.

Par contraste, il faut souligner qu'aussi bien au niveau municipal qu'autonomique, nous nous trouvons devant le pire résultat électoral du Parti Socialiste qui a perdu les élections dans des communes aussi emblématiques que Barcelone ou Séville, et, comme nous l'avons déjà indiqué, toutes les communautés autonomes qu'il gouvernait.

Après mai 2011, le PSOE ne se maintient qu'à la tête de sept chefs-lieux de province (Cuenca, Lugo, Ourense, Ségovie, Soria, Tolède et Saragosse), et le PSC deux (Lleida et Tarragone). Tous les autres restent ou passent aux mains du PP (34), de CiU (Barcelone et Gérone), du PNV (Bilbao) de Bildu-EA (Saint-Sébastien), de l'UPN (Pampelune), du BNG (Pontevedra) et de la Coalición Canaria (Sta. Cruz de Tenerife). Si nous prenons en considération les municipalités de plus de 75000 habitants, le PP a obtenu d'en gouverner 67, contre 21 au PSOE. En outre, au fur et à mesure que croît la taille de la commune, c'est la distance entre le PP et le PSOE qui se creuse. Si dans les municipalités de moins de 50000 habitants, l'écart est de $4 \%$ entre les deux formations, il est de $16 \%$ dans les villes de plus de 100000 . Le PSOE ne gouverne plus que l'Andalousie, où les élections autonomiques se dérouleront en mars 2012, et le Pays Basque, où il gouverne sans majorité avec l'appui parlementaire du PP...

La chute socialiste a donc été considérable, puisqu'en 2007 c'était l'équilibre qui prévalait entre les deux grands partis, avec un PP à la tête de 22 chefs-lieux provinciaux contre 23 au PSOE. Seules les élections de 1995, où le PP conquit 39 chefs-lieux contre 5 aux socialistes, peuvent être comparées à un tel renversement politique.

L'effondrement socialiste ne correspond pas non plus à un regain significatif de l'influence électorale de la gauche non socialiste. Izquierda UnidaIniciativa per Catalunya progresse d'à peine $0,8 \%$ par rapport à 2007 , mais reste très loin des résultats qu'elle avait obtenus pendant la période 19912003. Pour leur part, Esquerra Republicana de Catalunya comme le Bloque Nacionalista Galego perdent du terrain par rapport à 2007.

Les résultats des élections municipales de 2011 confortent l'implantation électorale du PP, consolident et renforcent son pouvoir local. La carte de son hégémonie électorale est claire, au-delà des conquêtes provinciales et de son 
nombre élevé de conseillers. Elle est nette dans une ample frange de territoire qui va de la Galice à la côte est, en occupant l'ensemble des zones centrales de la péninsule. Seuls le nord-nord/est et le sud-est conservent une couleur politique distincte, au profit du PSOE, de CiU ou du PNV (Ministerio de Interior, 2011). Les cartes 1 et 2 montrent de manière limpide la progression du PP entre 2007 et 2011 au niveau provincial, dans sa confrontation avec le PSOE-PSC. On se concentre sur la confrontation municipale entre socialistes et populaires, même s'il faut rappeler que dans certaines provinces, c'est un tiers parti qui l'emporte (CiU, PNV o UPN). Mais cela illustre bien l'avance acquise par le PP dans la confrontation bipolaire.

Cartes 1 et 2. Le parti victorieux du duel PP - PSOE/PSC
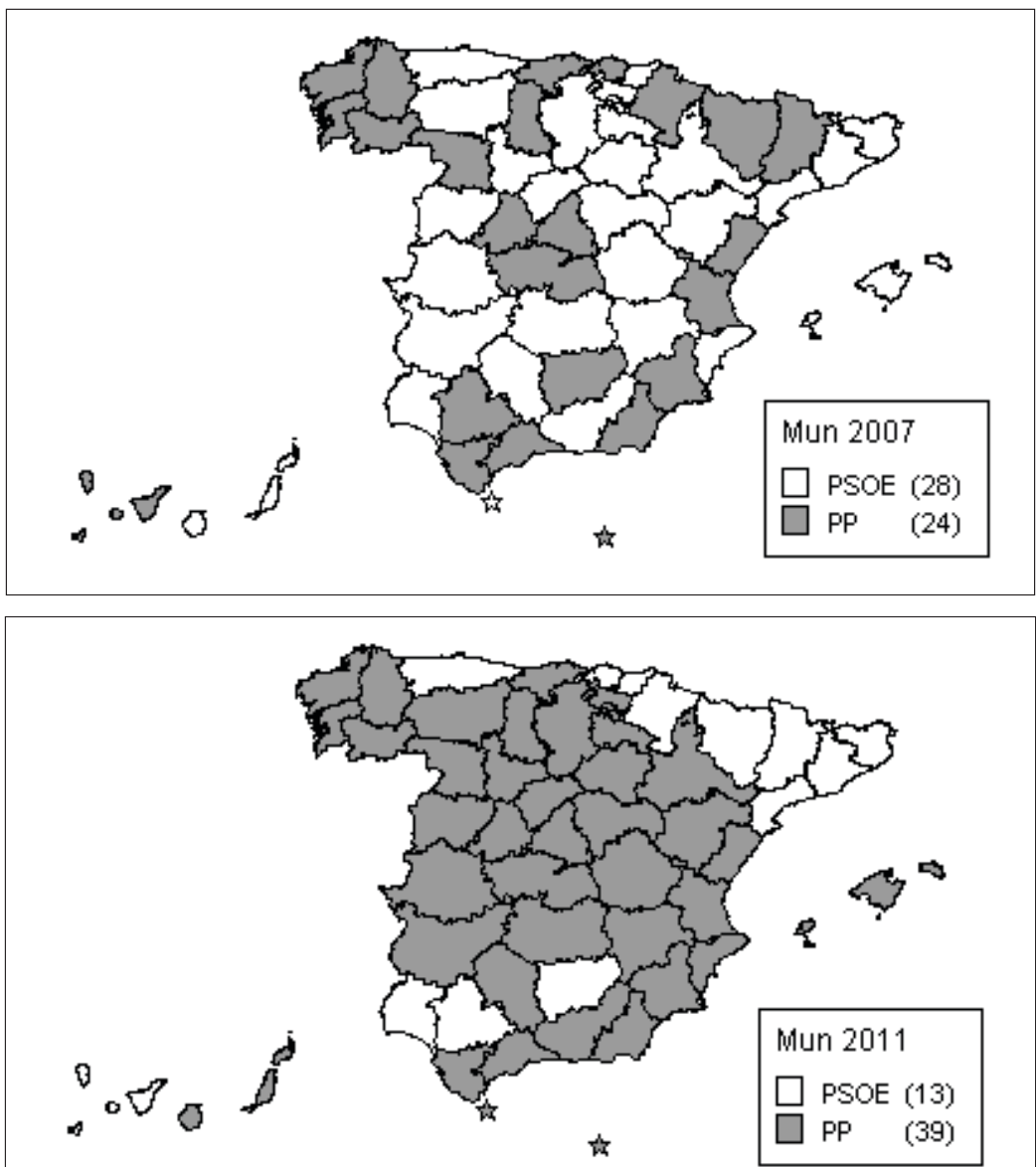
Dans le temps, ce résultat est comparable à celui de 1995, qui avait inauguré un cycle conservateur. Comme le montrent les tableaux 1 et 2 , qui présentent les résultats des élections municipales et ceux, agrégés, des élections autonomiques de 1991 à 2011, l'hégémonie détenue par le PSOE aux niveaux municipal et autonomique jusqu'au début des années 1990 s'achève brutalement en 1995, signe avant-coureur de la victoire du PP lors des élections législatives de 1996.

Le PP, qui s'était refondé sur les cendres de la vieille Allianza Popular de Manuel Fraga en 1989, avait déjà obtenu un léger regain de suffrages en 1991 (+ 5 \% par rapport aux élections précédentes de 1987). Il avait, entre autres, conquis le pouvoir municipal à Madrid et Valence, mais c'est en 1995 qu'il concrétisa son grand bond en avant, déjà annoncé par le bon résultat des élections européennes de 1994. Le PP dépassa de 5 points le PSOE sur l'ensemble du territoire espagnol, mais il augmentait son score de $10 \%$, tandis que le PSOE chutait de $8 \%$ par rapport à 1991. Avec cette victoire, le PP gouverne, ainsi qu'on l'a dit, une grande partie des capitales provinciales et gouverne 11 des 13 parlements régionaux, dont 5 avec une majorité absolue (Marcet 2010). Avec de tels résultats, c'est une nouvelle étape que franchit le $\mathrm{PP}$, qu'il soit ou non à la tête du gouvernement espagnol : il consolide son pouvoir municipal et régional lors des élections suivantes, jusqu'à celles de mai 2011, qui ont représenté un nouveau bond en avant et un renforcement notable de son hégémonie locale et régionale. Comme le montre le tableau 2 , alors que le PSOE a obtenu des résultats agrégés pour les élections autonomiques qui oscillent entre 6,6 millions de voix en 1995 et 8,6 millions en 2007, le PP amorce son avancée en 1995 en dépassant le PSOE de près d'un million et demi de voix, et n'a pas cessé de faire croître le nombre absolu de voix depuis ces élections, jusqu'à se rapprocher des 9 millions de voix en 2011, ce qui illustre sa capacité de progresser dans l'arène électorale autonomique.

Tableau 2. Votes et pourcentages agrégés du PSOE et du PP aux élections autonomiques (1991-2011)

\begin{tabular}{|c|c|c|c|c|}
\cline { 2 - 5 } \multicolumn{1}{c|}{} & \multicolumn{2}{c|}{ PSOE } & \multicolumn{2}{c|}{ PP } \\
\hline Année & Nombre de voix & $\begin{array}{c}\text { \% des suffrages } \\
\text { exprimés }\end{array}$ & Nombre de voix & $\begin{array}{c}\text { \% des suffrages } \\
\text { exprimés }\end{array}$ \\
\hline 1991 & 7.004 .556 & 38,2 & 4.957 .770 & 27,0 \\
\hline 1995 & 6.691 .384 & 30,6 & 8.174 .670 & 37,4 \\
\hline 1999 & 7.888 .816 & 35,5 & 8.185 .345 & 36,8 \\
\hline 2003 & 8.235 .299 & 35,7 & 8.650 .731 & 37,5 \\
\hline 2007 & 8.659 .088 & 37,2 & 8.711 .859 & 37,4 \\
\hline 2011 & 7.282 .204 & 31,3 & 8.983 .065 & 38,6 \\
\hline
\end{tabular}

Source : élaboration propre d'après données du Congrès des Députés 


\section{LES FACTEURS D'EXPLICATION DES RÉSULTATS DU 22 MAI}

La science politique espagnole a largement entériné le fait que les élections municipales, et en partie les élections autonomiques sont des scrutins de second rang, dont l'importance est moindre que les élections législatives qui conduisent aux charges gouvernementales. Elle a également constaté la dissolution progressive des différences entre configurations territoriales et entériné le concept de « nationalisation du vote » (Delgado, 2010, pp. 15-16), et ces deux tendances l'orientent vers des analyses électorales qui traitent de façon homogène des réalités locales et territoriales qui renvoient à des logiques politiques distinctes, en ne cherchant à dégager que des lignes d'interprétation les plus générales possibles.

$\mathrm{Si}$, dans les lignes qui précèdent, nous avons considéré l'ensemble espagnol comme un tout homogène, sans prendre en considération les circonstances locales et autonomiques qui pourraient expliquer avec plus de profondeur les résultats de chacune des consultations, il devient évident, au moment d'aborder les facteurs explicatifs, qu'il existe un degré notable de contagion ou d'influence du cadre politique national sur les élections locale et autonomique.

Leur situation dans le calendrier électoral, elle-même - la dernière année de mandat du gouvernement - semble les convertir en une sorte de "test de popularité" ou d'épreuve de vérité quant à l'état de santé de la majorité et de l'opposition nationale.

Ainsi, le parti de gouvernement obtient traditionnellement de pires résultats lors des élections municipales que lors des législatives, les premières matérialisant, comme les élections européennes, une tendance de caractère intermédiaire de vote sanction à l'égard de la majorité en place. La manifestation de ce vote sanction n'est cependant pas systématique : lors des élections locales qui eurent lieu pendant les périodes de gouvernement socialiste (1983-1991 et 2004-2007), le principal bénéficiaire des reculs du PSOE fut Izquierda Unida, alors qu'en 1995 et en 2011, c'est en effet le Parti Populaire qui profite de la perte d'appui électoral des socialistes, annonçant un nouveau cycle électoral et politique.

Il est incontestable que la politique nationale a pesé sur les élections du 22 mai 2011, à côté de facteurs plus locaux ou territoriaux, et sans oublier d'autres raisons que des analyses politiques plus récentes ont mises en lumière.

Nier l'impact de la crise économique et politique qui s'est abattue sur l'Espagne depuis le début 2008, pratiquement au moment des précédentes élections nationales (mars 2008) serait nier l'évidence. La chute de l'économie depuis la seconde moitié de 2008, la récession depuis fin 2008 au début 2010, la croissance du chômage qui s'établissait en février 2011 à plus de $20 \%$ (plus du double de la moyenne européenne) et touchait de façon aggravée les jeunes de moins de 25 ans (43\% selon les données Eurostat), de même 
que la perception par les citoyens d'une gestion déficiente de cette crise par le gouvernement Zapatero, ont marqué profondément les trois années qui ont précédé ces élections locales et autonomiques. Mais cette perception a été encore alourdie, de mai 2010 à fin 2011, quand le gouvernement a été conduit à prendre une série de mesures, que l'opinion publique et les médias ont majoritairement considérées comme prises sous la pression des autorités d'autres pays européens. Si elles ont préservé l'Espagne des interventions que d'autres pays européens ont subies, elles se sont traduites par des restrictions de dépenses, des réformes structurelles qui ont affecté de nombreux groupes sociaux (les travailleurs manuels, fonctionnaires, retraités, classes moyennes) et ont provoqué des mobilisations syndicale et citoyenne. L'exemple le plus spectaculaire de celles-ci est bien sûr le mouvement initié le 15 mai (sept jours seulement avant les élections), connu comme celui des « Indignados » qui, à travers l'occupation de places dans beaucoup de villes espagnoles, prétendaient exprimer son rejet de la situation économique et politique du pays en crise, présentant une large liste de revendications et d'alternatives destinées à améliorer la situation économique et la qualité de la démocratie en Espagne (et plus largement en Europe).

Le mouvement du 15-M a aussi catalysé un profond malaise politique que plusieurs indicateurs signalaient depuis quelques années. Ainsi, les baromètres du Centre d'Investigation Sociologique (CIS) montrent la progressive perte de crédit de la gestion gouvernementale entre avril 2008 et avril 2011 , et une perte de confiance dans le président du gouvernement, Rodríguez Zapatero, dont la cote de confiance chute de $46 \%$ en avril 2008 à $17,1 \%$ en avril 2011, selon les baromètres CIS n 2761 et 2885. Ces évolutions ne s'accompagnent pas d'un gain de crédibilité du principal parti d'opposition, le PP, ni de son leader, Mariano Rajoy, dont la cote baisse également pendant la même période : les mêmes baromètres du CIS établissent la cote de confiance du leader du PP à 20,5\% en avril 2008 et à 16,3\% en avril 2011. Cela incite à prendre en considération le fait, montré par plusieurs sondages, que la classe politique et les partis constituent le troisième problème de l'Espagne, après le chômage et la situation économique ${ }^{11}$.

La perte de confiance en Rodríguez Zapatero et en son gouvernement apparaît de façon plus claire dans les secteurs plus jeunes, étudiants, et ceux disposant d'un haut niveau d'études, de même qu'auprès des employés et personnels des services, selon les mêmes baromètres. Ceci incite à penser à un progressif abandon de l'électorat plus jeune et le plus proche des socialistes - un abandon équivalent, sinon supérieur à celui qui marque d'autres classes d'âge ou catégories socioprofessionnelles. Il convient d'ajouter à cela le constat d'une perte progressive du vote urbain, déjà entamée lors des élections de 1991, amplifiée en 1995, mais que celles de 2011 surpassent avec la perte emblématique de Barcelone et Séville. Le recul électoral du PSC en Catalogne, au profit de CiU, et du PSOE en Andalousie, au profit du PP deux bastions socialistes - semblent accréditer l'émergence d'un nouveau

1. Le baromètre 2885 du CIS chiffrait à $21,5 \%$ la considération de la classe politique et les partis politiques comme un problème de l'Espagne. 
cycle politique, que les prochaines élections générales du 20 novembre 2011 et les élections autonomiques andalouses, prévues en mars 2012, devraient confirmer si l'on en croit les sondages et enquêtes électorales.

Un dernier élément de réflexion peut compléter cette analyse des facteurs explicatifs des résultats de mai 2011. Si la crise économique et la perte de confiance dans le gouvernement qui la gère s'étendent à l'ensemble des partis au pouvoir dans la plupart des pays, il semble que le fait que la majorité y soit de gauche constitue un fait aggravant. Qu'il soit socialiste ou travailliste, on lui impute plus négativement le poids des mesures de lutte contre la crise financière qu'on ne le fait à l'égard de la droite gouvernementale. La démobilisation supérieure de la gauche en situation de crise, et le défaut d'alternative claire à l'orthodoxie économique seraient les causes d'une certaine désorientation programmatique et d'un déclin du leadership des options socialistes, face à l'éclosion de programmes conservateurs qui, en marge des qualités propres de leurs leaders, mobilisent plus fortement l'électorat de centredroit. C'est une situation qui a également conduit à une présence croissante, dans l'espace politique et électoral, d'options clairement populistes.

\section{LES ÉLECTIONS MUNICIPALES DE 2011 en Catalogne}

La science politique a l'habitude de distinguer les élections selon qu'elles penchent du côté du changement ou de la continuité d'un scrutin à l'autre. On évoque parfois la rupture, mais dans toute élection peuvent se trouver à la fois des éléments de continuité et des indices de changement. L'important est d'identifier ce qui prédomine, et l'importance des divers facteurs qui concourent à tel résultat, qui se puisse ranger plus ou moins du côté du changement ou de la continuité.

Dans le cas des élections municipales de 2011 en Catalogne, il est évident que les facteurs de changement l'emportent. De fait, le comportement électoral catalan est plongé dans un processus de changement qui dure déjà depuis plusieurs années. L'histoire électorale catalane, avec trente quatre scrutins au cours des 34 dernières années, a vécu une période assez longue de stabilité, qui a commencé après une première phase de constitution du système (qui aura duré jusqu'aux élections législatives de 1982), et qui s'est étendue jusqu'à la fin du siècle dernier. Plus ou moins vingt années de stabilité d'un modèle qui semblait solidement étayé, et qui avait fait baisser la garde aux chercheurs comme aux analystes. Les bases fondamentales de ce modèle auront acquis au gré des différents scrutins le statut de vérités incontestables, comme gravées dans du marbre.

Qu'étaient ces fondements ? Le système électoral catalan se basait sur la prééminence de deux partis centraux, celui de centre-droit nationaliste de CiU, et le Partit dels Socialistes de Catalunya (PSC), que se partageaient les faveurs de la majorité en fonction du type d'élection. Les socialistes ga- 
gnaient les élections législatives et municipales tandis que $\mathrm{CiU}$ remportait les autonomiques. Cette alternance des préférences électorales se basait sur deux mouvements simultanés : d'un côté, le transfert de voix d'un parti à l'autre d'un segment significatif d'électeurs en fonction du type d'élection et, de l'autre côté, l'abstention d'une partie de l'électorat penchant vers les socialistes lors des élections autonomiques, ce que l'on a ensuite appelé l' « abstention différentielle », liée à des éléments de compétence, d'importance (élection de second rang) et d'identité.

Les autres acteurs du système, dans ce modèle de comportement « classique », exerçaient un simple rôle de comparse, secondaire. Il est évident qu'au niveau local quelques unes de ces forces disposaient d'une présence significative (principalement Iniciativa per Catalunya Verds, l'ancien parti communiste), avec des bases électorales territorialisées dans les grandes villes autour de Barcelone.

Le seul changement substantiel de ce modèle intervint à partir des années 1990, quand le Parti Populaire devint le troisième parti du système au cours des élections législatives, au pic de son ascension espagnole, qui le propulsera au gouvernement en 1996. Les premiers signes d'épuisement du modèle se manifestent en 1999, quand une part significative du vote traditionnel de $\mathrm{CiU}$ s'abstient lors des élections municipales. Les nationalistes perdent 200000 voix par rapport aux élections de 1995. La même année, lors des élections autonomiques, se produit l'impensable : le PSC remporte les élections, avec toutefois une marge étroite de 5000 voix d'avances qui ne lui permettent pas de former un gouvernement.

Au cours des législatives suivantes, un autre élément imprévu intervient : le vote socialiste aux élections générales est inférieur à celui des élections autonomiques précédentes. Quelque chose se passait. Et ce quelque chose se matérialisa au cours du cycle électoral suivant, quand la part la plus substantielle du vote démobilisé du côté de CiU ne revient pas à son camp d'origine mais se dirige sciemment vers un appui à Esquerra Republicana de Catalunya (ERC), une formation ouvertement indépendantiste, qui multiple ses voix par deux lors des municipales et autonomiques, et par trois lors des élections générales de 2004. Définitivement, le système avait changé.

D'un modèle à deux partis principaux, on passa à un système où les deux partis secondaires ont une importance majeure, que ce soit dans le calcul des votes ou dans l'élaboration des coalitions de gouvernement. De fait, après les élections autonomiques de 2003, et en dépit du fait que le PSC ne dépassait $\mathrm{CiU}$ que de 8000 voix, se produisit l'alternance, principalement grâce à l'essor de ERC.

Le second cycle électoral suppose une certaine « correction » du précédent, puisque les indépendantistes de ERC subissent un recul significatif au cours de toutes les élections suivantes, des locales aux générales en passant par les autonomiques. Mais ici, on ne peut parler de retour au modèle « classique », mais d'une nouvelle phase d'alignement électoral dans un contexte de forte volatilité et d'une abstention rampante, que l'on a qualifiée de désaffection. La participation aux élections municipales en 2007 atteint 
son minimum historique $(53,8 \%)$, tandis que les élections autonomiques enregistrent leur second plus mauvais score.

Avec ces antécédents, l'élection municipale de 2011 se traduit par un pas de plus en terrain encore inconnu, qui associe des éléments du passé et des nouveaux, dont certains franchement préoccupants et inédits jusqu'alors dans la politique catalane.

Avant toute chose, c'est la première fois que CiU remporte les élections municipales. Jamais auparavant le nationalisme conservateur n'avait réussi à dépasser le PSC lors de ce type de scrutin. La victoire est encore plus nette si on tient compte du fait que $\mathrm{CiU}$ ravit aux socialistes le gouvernement de la métropole : Barcelone.

La victoire de $\mathrm{CiU}$ suit de près celle des élections autonomiques, six mois auparavant, même si les élections municipales ne conduisent pas à une augmentation des suffrages nationalistes. Ce sont les socialistes, avec une chute importante (200000 voix de moins qu'en 2007), qui favorisent le triomphe de CiU. Cette perte de soutien au PSC se rapproche non seulement de celle enregistrée lors des élections municipales entre 2003 et 2007 (environ 180000 voix de moins), mais de celle des élections autonomiques entre 2006 et 2010 (200 000 voix de moins, qui s'ajoutent aux 230000 perdues entre 2003 et 2006). Sur l'ensemble des deux derniers cycles électoraux, les socialistes ont perdu plus de $40 \%$ de leurs voix aux élections autonomiques, et le tiers de leur capital électoral municipal.

Le déclin du PSC, s'ajoutant à l'affaiblissement du vote CiU aux élections municipales, fait que le poids des deux principaux partis du système se situe désormais à environ $50 \%$ des voix. C'est un élément fondamental de changement de modèle. Il y a vingt ans, lors de l'élection de 1991, le total des voix PSC + CiU atteignait $70 \%$ du total des suffrages exprimés. Vingt ans plus tard, le poids des deux grands a chuté de $20 \%$. Le système s'est fait plus pluriel, moins bipartisan.

Cet essor du pluralisme est le second élément à souligner, dans la mesure où ce n'est pas seulement le fait que les deux « grands » aient perdu de l'influence, mais aussi que la liste des « petits » partis se soit étendue. Aux traditionnels PP, ICV et ERC se sont ajoutées de nouvelles formations qui semblent capables de s'approprier un espace politique spécifique. Parmi elles on peut distinguer Ciutadans, une formation dont l'objectif principal est la défense de la langue castillane en Catalogne, et qui est parvenue à conserver en 2010 la représentation parlementaire qu'il avait obtenue en 2006 (3 députés catalans). À l'autre extrême du spectre politique se situent des formations qui profitent de la déconfiture de ERC, dont certaines disposent d'une représentation parlementaire dès 2010 (Solidaritat per la Independència, 4 députés), et d'autres bénéficient d'une base locale importante, comme les Candidatures d'Unitat Popular, qui lors de ces élections municipales ont multiplié par 4 le résultat obtenu en 2007. Enfin, il faut mentionner la formation xénophobe Plataforma per Catalunya, qui multiplie par 5 son score de 2007, et manque de peu son entrée au Parlement en 2010. 
Ainsi donc, on assiste à l'affaiblissement des acteurs principaux, la prolifération, avec succès, d'offres aux extrêmes du système et la croissance exponentielle d'un vote de rejet, exprimé par le biais du vote blanc et, lors de ce scrutin, du vote nul, encouragé par les protestations citoyennes du mouvement des «Indignados ». Le vote blanc avait déjà montré sa capacité d'attirer le mécontentement lors des élections de 2007, où il augmenta de $60 \%$. Désormais, au lieu de diminuer, il a continué à croître pour atteindre près de 120000 bulletins. La croissance du vote nul est circonscrite à cette élection, avec une augmentation de 30000 bulletins par rapport à 2007 .

Dans l'attente de ce qui se passera lors des élections générales du 20 novembre 2011, qui parachèveront l'actuel cycle électoral, une chose semble claire : les vérités immuables qui fondaient l'explication du vote en Catalogne ne sont déjà plus capables de donner une explication globale et certaine de ce qui se passe. Il conviendra de chercher de nouveaux outils pour définir et comprendre le comportement électoral catalan, chaque fois plus éloigné des références canoniques du modèle « classique ».

Tableau 3: Résultats des élections municipales en Catalogne 1991-2011 (en $\%$ des suffrages exprimés)

\begin{tabular}{|l|c|c|c|c|c|c|}
\cline { 2 - 7 } \multicolumn{1}{c|}{} & $\mathbf{1 9 9 1}$ & $\mathbf{1 9 9 5}$ & $\mathbf{1 9 9 9}$ & $\mathbf{2 0 0 3}$ & $\mathbf{2 0 0 7}$ & $\mathbf{2 0 1 1}$ \\
\hline Participation & 57,7 & 64,9 & 55,5 & 61,3 & 53,8 & 55,3 \\
\hline Blancs & 0,7 & 0,9 & 1,1 & 1,0 & 1,7 & 2,3 \\
\hline Nuls & 0,3 & 0,3 & 0,4 & 0,3 & 0,4 & 1,0 \\
\hline & & & & & & \\
\hline PSC & 37,1 & 32,9 & 37,3 & 34,0 & 32,0 & 24,7 \\
\hline CiU & 33,0 & 29,7 & 26,1 & 24,0 & 25,0 & 26,7 \\
\hline PP & 6,7 & 12,2 & 10,9 & 11,1 & 9,8 & 12,5 \\
\hline IC & 9,3 & 11,8 & 7,5 & 10,3 & 9,0 & 8,2 \\
\hline ERC & 3,4 & 6,2 & 7,6 & 12,7 & 11,6 & 8,8 \\
\hline Autres & 8,9 & 5,2 & 7,8 & 5,6 & 8,8 & 13,3 \\
\hline
\end{tabular}

Source : Élaboration propre d'après données du Ministère de l'Intérieur 


\section{RÉFÉRENCES / REFERENCES}

Delgado I., El comportamiento electoral municipal español, 1979-2005, Madrid, Centro de Investigaciones Sociológicas, 1997.

Delgado I., « Elecciones Municipales en España. Dimensiones analíticas y aspectos distintivos de ocho procesos electorales (1979-2007) », Política y Sociedad, vol. 47, $\mathrm{n}^{\circ} 3,2010$.

Marcet J., Où en est la droite ?: l'Espagne, Paris, Fondapol, 2010.

Ministerio del Interior, page web pour cartographie : www.mir.es, consulté le 3 octobre 2011

Urquizu I., « El vulco electoral del 22-M », Claves de la razón práctica, n² 214, 2011. 